\title{
Long-term use of adalimumab in the treatment of moderate to severe plaque psoriasis: a review of the literature
}

This article was published in the following Dove Press journal:

Clinical, Cosmetic and Investigational Dermatology

17 April 2010

Number of times this article has been viewed

\author{
Angela Y Moore \\ Blakely S Richardson \\ Arlington Center for Dermatology, \\ Arlington, Texas, USA
}

\begin{abstract}
Psoriasis is a chronic T-cell-mediated inflammatory disease that primarily affects the skin and joints. Patients with moderate to severe psoriasis constitute about $30 \%$ of the psoriasis population. Treatment of this group is challenging due to the long-term side effects, toxicities and inconvenience of conventional treatments such as phototherapy, methotrexate and cyclosporine. However, recent advances in our understanding of the pathogenesis of psoriasis have led to the popular use of biologics, which offer a safer, more convenient and effective targeted therapy. Adalimumab was originally approved for treating rheumatoid arthritis. Currently, adalimumab is also approved for treatment of adult patients with moderate to severe chronic plaque psoriasis who are candidates for systemic therapy or phototherapy or when other systemic therapies are medically less appropriate. Since the onset of the use of biologics, there have been concerns over safety and efficacy when used as long-term therapy. This paper reviews all publications, posters and abstracts reporting original data on the efficacy and/or safety of adalimumab in patients treated for chronic plaque psoriasis for more than 1 year.
\end{abstract}

Keywords: psoriasis, adalimumab, biologics

\section{Introduction}

Psoriasis is a chronic T-cell-mediated inflammatory disease that primarily affects the skin and joints with an estimated world-wide prevalence of 3\%. Accurate diagnosis is important due to recent evidence that psoriasis may be associated with other co-morbidities such as metabolic disorders, obesity and may be an independent risk factor for cardiovascular disease. ${ }^{1}$ The most common form of psoriasis is plaque psoriasis, which constitutes around $80 \%$ to $90 \%$ of psoriasis patients. Other less common forms include inverse, erythrodermic, pustular, and guttate psoriasis. ${ }^{2}$

Psoriatic arthritis (PsA) is an inflammatory arthritis characterized by pain, swelling and stiffness of the joints that affects between $6 \%$ to $42 \%$ of patients with psoriasis. Joint symptoms often precede skin manifestations of the disease by an average of 10 years. $^{3}$

Patients with moderate to severe psoriasis constitute about $30 \%$ of the psoriasis population. These patients are determined by a score greater than 10 on several indices including: total body surface area (TbSA 1\%-100\%), psoriasis area and severity index (PASI 0-72), and dermatology quality of life index (DQLI 1-30). This subset of patients presents a challenge due to the long-term side effects, toxicities and inconvenience of conventional treatments such as phototherapy, methotrexate and cyclosporine. However, recent advances in our understanding of the pathogenesis of psoriasis have led to the popular use of biologics, which offer a safer, more convenient and effective targeted therapy. ${ }^{4}$
Correspondence:Angela Y Moore Arlington Center for Dermatology, 7II East Lamar Blvd., Suite 200 Arlington, Texas 760II, USA Email acdermatology@yahoo.com 
Recently, several studies suggest that patients with severe psoriasis may have an increased risk for cardiovascular disease, depressive illness, lymphoma (Hodgkin's lymphoma and cutaneous T-cell lymphoma), and a decreased life expectancy. ${ }^{5-10}$

We systematically reviewed all published articles, posters, and abstracts reporting original data concerning the efficacy and/or safety of adalimumab in chronic plaque psoriasis for more than 1 year. Relevant articles were identified by searching Medline from its inception until December, 2009 for combinations of the search terms "psoriasis" and "adalimumab". Animal studies were excluded. No persons other than the authors of this manuscript were involved in the design, analysis, and interpretation of data, in the writing of the report, and in the decision to submit the article for publication.

\section{Pathogenesis of psoriasis}

The pathogenesis of psoriasis is currently viewed as a disorder of TH1 cell-mediated immunity. Tumor necrosis factor $\alpha$ (TNF- $\alpha$ ), which is a natural occurring cytokine, is elevated in the plaques as well and in the synovial joints and serum in psoriasis patients. TNF- $\alpha$ appears to be responsible for the keratinocyte hyperproliferation, endothelial cell regulation, and recruitment and activation of memory $T$ cells that perpetuate the inflammatory response in psoriasis. ${ }^{7} \mathrm{TNF}-\alpha$, produced by activated $\mathrm{T}$-cells, promotes the inflammatory response by activating nuclear factor $\mathrm{\kappa B}$, leading to the induction of interleukin (IL1)/6/8 and to the up-regulation of adhesion molecules on endothelial cells. These interleukins are responsible for the up-regulation of adhesion molecules on endothelial cells. ${ }^{4}$ IL-23 has also been found in increased levels and stimulates the survival and proliferation of TH17 cells. More research is needed to access the role in the TH17 pathway in the pathogenesis of psoriasis, as it may play a key role in effective treatment options. ${ }^{8}$

\section{Anti-TNF agents}

Anti-TNF- $\alpha$ agents block the inflammatory effects of TNF- $\alpha$ and include adalimumab, infliximab, and etanercept. Blocking TNF- $\alpha$ may also inhibit the Th17 pathway. These TNF agents have become popular treatment options for a variety of inflammatory disorders including psoriasis and PsA. ${ }^{4}$

\section{Pharmacology and recommended dosing of adalimumab}

Adalimumab is the first fully humanized recombinant monoclonal antibody that is indistinguishable in structure and function from naturally occurring IgG1. Adalimumab binds to both soluble and cell-bound TNF- $\alpha$ and prevents TNF- $\alpha$ interaction with the $\mathrm{p} 55$ and $\mathrm{p} 75$ cell surface TNF receptors. Adalimumab lyses surface TNF expressing cells in vitro in the presence of complement and also induces apoptosis in cells expressing surface TNF- $\alpha$, thereby neutralizing its effects and improving joint and skin manifestations. ${ }^{12}$ In addition, adalimumab modulates biological responses that are induced or regulated by TNF, including changes in the levels of adhesion molecules responsible for leukocyte migration (ELAM-1, VCAM-1, and ICAM-1 with an $\mathrm{IC}_{50}$ of $\left.1-2 \times 10^{-10} \mathrm{M}\right) .^{12}$

According to the therapeutic window concept, ${ }^{9}$ the steadystate range of serum or tissue drug concentrations should be adequate for neutralization of surplus TNF, but not so high as to threaten safety because of neutralization of homeostatic concentrations of TNF required for host defense, or so low as to impair efficacy from suboptimal neutralization of TNF. Recommended dosing for the treatment of psoriasis is $80 \mathrm{mg}$ given subcutaneously at week one, $40 \mathrm{mg}$ given subcutaneously at week 2 , followed by regular dosing of $40 \mathrm{mg}$ subcutaneously administered every 2 weeks thereafter. ${ }^{10}$ Adalimumab given $40 \mathrm{mg}$ subcutaneously every other week produces steady-state serum trough concentrations of 4 to $8 \mu \mathrm{g} / \mathrm{mL}$, which are 3 to 7 times greater than the clinically effective serum concentrations $(0.8-1.4 \mu \mathrm{g} / \mathrm{mL}) .{ }^{11}$ The systemic clearance of adalimumab is approximately $12 \mathrm{~mL} / \mathrm{h}$. Once therapy is initiated, the drug reaches its peak around 5.5 days and has a half-life of approximately 14 days ranging from 10 to 20 days across studies. Mean serum adalimumab trough levels at steady state increases proportionally with dose following 20, 40, and $80 \mathrm{mg}$ every other week and every week subcutaneous dosing. ${ }^{10}$ In long-term studies with dosing more than 2 years, no evidence exists of changes in clearance over time.

\section{Current approval}

Adalimumab is currently Food and Drug Administration (FDA) and European Medicines Agency (EMEA) approved for the treatment of PsA, rheumatoid arthritis (RA), Crohn's disease (CD), juvenile idiopathic arthritis (JIA), ankylosing spondylitis (AS), and, more recently, moderate to severe plaque psoriasis. ${ }^{10}$ Specifically, in the US, adalimumab is approved for treatment of adult patients with moderate to severe chronic plaque psoriasis who are candidates for systemic therapy or phototherapy or when other systemic therapies are medically less appropriate. Before starting treatment with adalimumab, a complete history and physical exam should be performed. Recommended labs include a complete 
blood cell count, chemistry panel with liver function tests, hepatitis panel, and tuberculosis $(\mathrm{Tb})$ testing at baseline and at variable frequencies thereafter. ${ }^{14}$

Limited safety experience exists on surgical procedures in patients treated with adalimumab. The long half-life of the drug should be taken into consideration, and a patient requiring surgery should be closely monitored for infections. Patients on adalimumab may receive concurrent vaccinations, except for live vaccines. No data currently exist on secondary transmission of infection by live vaccines in patients receiving adalimumab therapy. ${ }^{16}$ Adalimumab did not diminish response to pneumococcal and influenza virus vaccines in RA patients. ${ }^{17}$

Adalimumab is a pregnancy category B drug. ${ }^{18}$ Because of the possible impact on newborn's immune responses, adalimumab treatment in women of reproductive age requires contraception during treatment and at least 5 months after the last administered dose. Women should not breast feed for at least 5 months after the last adalimumab treatment. ${ }^{14}$

Patients with RA treated with adalimumab during the first trimester of pregnancy were followed by the Organization of Teratology Information Specialists (OTIS) throughout pregnancy and for 1 year post partum and compared with those from a disease-matched group of pregnant women with RA without adalimumab treatment during pregnancy, and a healthy (ie, non-diseased) group of women who neither had RA nor had been treated with adalimumab. ${ }^{18}$ In addition, OTIS investigators collected information on patients with psoriasis or other diseases treated with adalimumab. Based on these preliminary data, no increases in major structural defects or major malformations were seen in women with adalimumab exposure. The following perinatal complications were observed: Prematurity, neonatal jaundice, neonatal urinary Escherichia coli infection, and adrenal congenital hyperplasia of probable hereditary origin. However, more recently, data that had been reported to the FDA on children of pregnant mothers taking a TNF- $\alpha$ antagonist have uncovered a more significant increase in congenital anomalies consistent with those seen in VACTERL association. ${ }^{19}$ Based on these data, we agree with the suggestion that clinicians should probably not prescribe TNF antagonists to women during pregnancy since human experience is still extremely limited, particularly in patients with psoriasis.

\section{Efficacy and safety concerns based on previous RA studies}

To date, most large-scale safety assessments of adalimumab are based on trials of patients with RA. Patients with RA are often treated with the combination of TNF inhibitors and an immunosuppressive agent such as methotrexate, while patients with psoriasis are often treated with the TNF inhibitors as monotherapy. The extrapolations on the safety of TNF inhibitors derived from this combination therapy data may actually overestimate the potential risk of these agents when used as monotherapy in psoriasis. ${ }^{14}$ It is important to be aware of potential adverse events that have been reported with the use of adalimumab in RA studies. Serious adverse events of concern are discussed below. However, the safety data based on RA studies should only be cautiously compared between other indications such as moderate to severe plaque psoriasis, due to differences in co-morbidities and patient populations.

Studies of RA patients have shown that the most common adverse events associated with adalimumab therapy are injection-site reactions. They are usually mild and include local erythema and pruritus. In clinical studies, 53\% of adalimumab patients with RA developed infections compared with $47 \%$ of placebo-treated patients. Other common adverse events include upper respiratory tract infections, rhinitis, bronchitis and urinary tract infections. ${ }^{16}$

The incidence of serious infections in studies of RA patients was under $2 \%$. However, patients with underlying predisposing medical conditions are at a higher risk. Rare opportunistic infections, including histoplasmosis, cryptococcosis, aspergillosis, coccidioidomycosis, candidiasis, listeriosis and pneumocystis, have been reported. Most of these patients were also treated with other immunosuppressive agents, such as methotrexate, systemic corticosteroids, or both. ${ }^{16}$

In the event of an infection requiring antibiotic therapy, adalimumab should be withheld and appropriate treatment should be initiated. However, in the event of more serious infections or opportunistic infections, adalimumab should be discontinued and the patient should be treated appropriately. ${ }^{20}$ Treatment with TNF inhibitors should be avoided if possible in patients with chronic, serious, or recurring infections. ${ }^{14}$

Registry data from patients with RA and post marketing reports to the FDA have identified numerous cases of $\mathrm{Tb}$ reactivation associated with all TNF inhibitors. ${ }^{14}$ Extrapulmonary or disseminated cases of $\mathrm{Tb}$ occurred in 7 patients on anti-TNF therapy during the first 534 patientyears of adalimumab exposure in clinical trials; the rate of $\mathrm{Tb}$ decreased by $75 \%$ in European trials after the introduction of routine $\mathrm{Tb}$ screening. The FDA currently recommends $\mathrm{Tb}$ screening with a purified protein derivation before beginning anti-TNF therapy. ${ }^{16}$ A tuberculin skin test (PPD) or quantiferon Gold serum assay can be used to screen for Tb. ${ }^{14}$ For patients about to initiate anti-TNF therapy, a positive test 
is read if greater than $5 \mathrm{~mm}$ induration is observed at 48 to 72 hours. For a positive PPD reading, a follow-up chest Xray is indicated to rule out an active infection. If a patient is diagnosed with a latent infection, prophylaxis with 9 months of isoniazid is warranted. Anti-TNF therapy may be initiated after 1 to 2 months only if the patient is adhering to and tolerating the isoniazid therapy. Patients with active $\mathrm{Tb}$ should be appropriately referred to a specialist to begin the standard 4-drug treatment. Concurrent anti-TNF therapy is contraindicated in active $\mathrm{Tb}{ }^{20}$

In trials of patients with RA treated with adalimumab, the incidence rate of lymphomas was 3.1. Risk of lymphoma is substantially increased in a subset of patients with very severe RA, regardless of previous treatment types. High inflammatory activity, rather than its treatment, is a major risk determinant. ${ }^{21}$ Further investigation is warranted to evaluate if TNF inhibitors are associated with increased lymphoma rates.

Patients treated with adalimumab tend to have a higher incidence of demyelinating conditions, especially multiple sclerosis (MS). MS has been reported to develop in and also worsen in patients taking TNF inhibitors. However it has been suggested that psoriasis and RA patients have a higher incidence of MS compared to the general population. ${ }^{16} \mathrm{TNF}$ inhibitors should be avoided in the setting of a personal history of demyelinating conditions or patients with first-degree relatives with $\mathrm{MS} .{ }^{14}$

To determine the hazard risk of developing or worsening heart failure, RA patients treated with infliximab, etanercept, or adalimumab $(n=2757)$ or conventional disease-modifying anti-rheumatic drugs (controls; $\mathrm{n}=1491$ ) in a German biologics register were studied. ${ }^{22}$ No evidence was found of an increased risk of heart failure with TNF- $\alpha$ inhibitors, especially if no concomitant treatment with glucocorticoids or cyclooxygenase 2 inhibitors is given. Since exacerbation of chronic heart failure (CHF) has been reported with TNF inhibitors, however, it has been recommended that adalimumab be avoided in patients with severe CHF (class III or IV) and discontinued in those with mild disease during a CHF exacerbation. ${ }^{23}$

Data suggest there may be a risk of hepatotoxicity and hepatitis $B$ reactivation in adalimumab patients. Whereas TNF- $\alpha$ may promote viral clearance in hepatitis $B$, it is thought to promote chronic liver injury in hepatitis $\mathrm{C}$, with elevated levels of TNF- $\alpha$ in patients with hepatitis C compared to the general population. A few reports have stated that anti-TNF treatment in hepatitis $\mathrm{C}$ is safe and well tolerated by patients and may even be beneficial. ${ }^{24}$ Consultation with liver specialists as indicated may be appropriate when considering the use of anti-TNF therapy in this setting. There is an FDA warning suggesting that patients who have concurrent hepatitis B infection should not be treated with any of the TNF inhibitors. ${ }^{14}$

The safety and efficacy of adalimumab in immunosuppressed patients have not been evaluated. HIV is listed among the relative contraindications for anti-TNF therapy. Because clinical trials have excluded patients with HIV/AIDS, limited data are available. However, in a study of 64 RA patients there was no evidence of depression of delayed-type hypersensitivity, depression of immunoglobulin levels or change the number of effector $\mathrm{T}$ and $\mathrm{B}$ cells, natural killer cells, monocytes, macrophages or neutrophils. There have been several case reports of HIV patients treated with etanercept and infliximab in which there did not appear to be an increase in morbidity or mortality. ${ }^{16}$ In some patients, some symptoms of HIV/AIDS such as aphthous ulcers, cachexia, dementia, and fatigue actually improved with TNF blockade. HIV/ AIDS patients who are well controlled on antiviral therapy and have failed all other treatment options may be carefully selected for anti-TNF therapy under careful surveillance of their physician. ${ }^{24}$ However, there are no conclusive data and no specific recommendations in the current guidelines.

In a prospective cohort study, in which 289 RA patients on anti-TNF therapy were followed through the course of several years, 128 dermatological events were recorded in 72 patients. ${ }^{12}$ In placebo-controlled randomized clinical trials on adalimumab in RA patients, conversion rates of patients with negative ANA at baseline to positive ANA occurred in $11 \%$ to $26 \%$ of RA patients treated with adalimumab, and $5.5 \%$ to $15.2 \%$ of RA patients who received placebo. ${ }^{26,27}$ Interestingly, $5 \%$ to $44 \%$ of RA patients converted from $\mathrm{ANA}(+)$ to ANA(-). Presenting manifestations were mostly cutaneous lesions, photosensitivity and serositis. ${ }^{27}$ No evidence exists that developing ANA, anti-DNA antibodies or anticardiolipin antibodies while on TNF antagonist therapy increases the risk of developing clinical systemic lupus erythematous (SLE)-type syndromes. ${ }^{28}$

However, one case of clinical SLE and 2 cases of cutaneous lupus have been identified in patients shortly after initiating adalimumab in RA patients in clinical practice. VanRijthoven et al described clinical symptoms of SLE in a 56-year-old female with RA who was loss response to infliximab so then switched to adalimumab. ${ }^{29}$ ANA and anti-dsDNA were positive at the initiation of adalimumab therapy and clinical symptoms of lupus (no organ involvement) ensued after 4 injections of adalimumab. Resolution of 
symptoms occurred after treatment with IV dexamethasone and adalimumab was discontinued. Spillane et al described a 40-year-old female with RA who developed biopsy-proven discoid lupus after her second to fourth doses of adalimumab. ${ }^{30}$ The patient had previously completed an 8-month course of etanercept but had switched to adalimumab. ANA and extranuclear antibodies panels were negative. Adalimumab was discontinued and the patient was restarted on etanercept 1 month later. No new lesions developed, and the existing lesions resolved with topical steroids. Sheth et al reported a 51-year-old female who developed a rash 10 days after starting adalimumab (40 mg every other week) for seropositive RA. ${ }^{31}$ She developed biopsy-proven subacute cutaneous lupus erythematosus on her arms and chest but no systemic symptoms, neurological symptoms, nor serositis. Symptoms resolved without treatment 2 weeks after discontinuing adalimumab. Family history was positive for SLE with renal and cutaneous involvement.

\section{Efficacy of adalimumab in the long-term treatment of moderate to severe plaque psoriasis and psoriatic arthritis}

Even though adalimumab has only been evaluated for up to 100 weeks for chronic plaque psoriasis, the efficacy and safety of adalimumab therapy has been evaluated up to 120 weeks for PsA. ${ }^{32}$ The ADEPT trial evaluated the long-term efficacy and safety in PsA over a 2-year period. Of particular interest is the efficacy of adalimumab in the clearance of skin lesions as well as the long-term safety profile. It is important to note that patients who were on non-steroidal anti-inflammatory agents, disease-modifying anti-rheumatic drugs or prednisone before the trial could continue these medications throughout the study. In this placebo-controlled trial, 298 patients completed a 24-week double-blind period; 285 patients were then enrolled in an open-label extension study and received $40 \mathrm{mg}$ adalimumab subcutaneous every other week for up to an additional 120 weeks. Skin assessments were restricted to patients with at least 3\% body surface area involvement at enrollment. Notably, greater than $20 \%$ achieved and maintained a PASI 100 at 48 weeks and $56.6 \%$ had a physicians global assessment (PGA) of clear or almost clear at 104 weeks. The percentage of patients achieving PASI 50, PASI 75, PASI 90, or PASI 100 changed by $10 \%$ or less from week 48 through 2 years of adalimumab treatment. Skin-related improvements that were present after 48 weeks of adalimumab therapy were maintained through 2 years of therapy for most patients. ${ }^{32}$
To compare treatment effectiveness as well as patient satisfaction and tolerability of the TNF- $\alpha$ antagonists, Heiberg et al compared the discontinuation rates of adalimumab, infliximab, and etanercept in patients with PsA, RA, and AS. ${ }^{33} 150$ patients were treated with 172 antiTNF- $\alpha$ treatment courses in patients with PsA (adalimumab $\mathrm{n}=28$; infliximab $\mathrm{n}=48$; etanercept $\mathrm{n}=96$ ). Concomitant methotrexate therapy (68\%) was associated with lower withdrawal rates of all three anti-TNF $\alpha$ agents. Discontinuation rates were lower for adalimumab (14.3\%) than for infliximab $(25.0 \%)$ or etanercept $(24.0 \%)$. These data suggest that $86 \%$ of patients treated with adalimumab maintained acceptable efficacy, patient satisfaction, and tolerability to maintain compliance.

In phase II double-blind, randomized controlled clinical trials of adalimumab in chronic plaque psoriasis, in the openlabel extension arm, patients who received adalimumab for 60 weeks for plaque psoriasis achieved PASI 75 in 56\% of the every other week, $64 \%$ of the every week, and $45 \%$ of the placebo/eow groups. Total clearance (PASI 100) at week 60 was observed in $16 \%$ of the every other week group, $26 \%$ of the every week group and $19 \%$ in the placebo/every other week group. ${ }^{34}$

In a phase III double-blind, randomized-controlled clinical trials of adalimumab $40 \mathrm{mg}$ every other week in 1212 patients with moderate-to-severe plaque-type psoriasis, Menter et al reported initial PASI-75 response rates of $71 \%$ after 16 weeks and $70 \%$ after 24 weeks of treatment. ${ }^{35}$ Patients who had met the PASI-75 response criterion were re-randomized at week 33 to continue treatment with adalimumab or switched to placebo until week 52. Loss of response over the 19 weeks, defined as $<$ PASI 50 and $\geq 6$ point increase in PASI, was observed in $5 \%$ of patients continuing adalimumab therapy and $28 \%$ of patients switched to placebo. Based on these findings, loss of response after continuous treatment with adalimumab over 1 year was 5\% while sustained efficacy was seen in $72 \%$ of patients for 19 weeks after discontinuation of adalimumab treatment.

A small retrospective study by Van et al evaluated 49 patients with moderate-to-severe plaque psoriasis who has lack of or loss of efficacy with prior biologic therapy including infliximab and/or etanercept. After 12 months of adalimumab therapy, $78 \%$ of patients were considered as "clear" or "almost clear" according to PGA of disease severity. Five patients (10\%) stopped adalimumab due to lack of effectiveness. ${ }^{36}$ Thus, it appears that prior treatment with another TNF- $\alpha$ antagonist does not significantly appear to diminish response to adalimumab. 
Although loss of efficacy over time has been observed in patients receiving infliximab, this does not appear to be an issue for adalimumab based on published data. ${ }^{37}$ In contrast, efficacy in patients with psoriasis treated continuously with adalimumab for 100 weeks sustained PASI 75 responses in $83 \%$ and PASI 100 responses in 32\%, with a PGA score of "clear" or "minimal" in $70 \% .{ }^{38}$ Similar efficacy was seen in patients with moderate to severe plaque psoriasis and PsA treated with adalimumab for 2 years. ${ }^{39}$

Mentor et al also assessed for the presence of antiadalimumab antibodies (AAAs). ${ }^{35} 8.8 \%$ had detectable AAAs during the 52-week treatment period. Three of 7 AAA-positive patients, compared with 65 of 233 AAAnegative patients, lost an adequate response. The presence of AAAs was not correlated with any treatment-related adverse events, including serious adverse events and injection-site reactions. Some patients who developed AAAs demonstrated reduced efficacy. However, because the percentage of patients who developed antibodies to adalimumab was small, the overall involvement of AAAs in reduction of efficacy was minimal. ${ }^{35}$

\section{Safety and tolerability of adalimumab in the long-term treatment of plaque psoriasis and psoriatic arthritis}

The safety profile of adalimumab in phase II clinical trials during long-term treatment of PsA and psoriasis was consistent with the safety profile of previous adalimumab studies. ${ }^{32}$ No clinically meaningful changes in the type and frequency of adverse events were observed with 24 versus 48 weeks of adalimumab treatment. No significant changes in laboratory values were seen, besides the desired effect of a decrease in $\mathrm{C}$-reactive protein. No new safety signals were seen compared to rheumatoid arthritis. Specifically, no indication of an increase in such TNF-associated adverse events such as Tb, malignancies, demyelinating disease, CHF, or SLE were observed. Through 2 years of adalimumab exposure, only 1 report of peritoneal Tb, 4 of oral candidiasis, 1 of non-Hodgkin's lymphoma, 2 of basal cell carcinoma, and 1 with a neuroendocrine carcinoma of the skin occurred. Three deaths occurred among the 298 patients. Two patients experienced cardiopulmonary arrest from a myocardial infarction, which was probably not related to adalimumab. The third patient experienced sudden death that was attributed to acute pulmonary edema and was considered to be possibly related to adalimumab. ${ }^{32}$

In phase-II clinical trials on patients receiving adalimumab for 60 weeks, adalimumab was generally well tolerated, with $9 \%$ of patients discontinuing therapy due to an adverse event. No significant laboratory abnormalities were observed. The most frequently reported side effects included nasopharyngitis and upper respiratory infection as well as pain at the injection site. Fourteen patients experienced a serious adverse event during the 60 -week period, being more common in the patients receiving adalimumab weekly (11 patients). Serious adverse events included 2 cases of malignant melanoma, 1 case of squamous cell carcinoma, 2 cases of cerebrovascular events, 1 case of breast carcinoma, 1 case of gastric adenocarcinoma, 1 case of coccidiomycosis and 2 cases of $\mathrm{Tb} .{ }^{34}$

Burmester et al performed a safety analysis in 19, 041 patients exposed to adalimumab using data from 36 global clinical trials though April 15, 2007, and concluded that the overall malignancy rates and the all-cause mortality rates for adalimumab treated patients were as expected for the general population..$^{40}$ Safety data were included from adalimumab stud-

Table I Compiled safety data (rates per 100 patient-years) for serious adverse events for adalimumab in different FDA-approved indications

\begin{tabular}{|c|c|c|c|c|c|c|}
\hline \multicolumn{7}{|c|}{ Established safety profile for adalimumab } \\
\hline Indication & Ps & PsA & RA & AS & JIA & CD \\
\hline Exposure (PY) & 135 & 484 & 12,506 & 423 & 99 & $\mathrm{I}, 506$ \\
\hline Patients (N) & 142 & 395 & 10,050 & 393 & $17 \mid$ & 1,459 \\
\hline Serious infections & 0.74 & 2.07 & 5.05 & 1.18 & 4.04 & 5.98 \\
\hline Tuberculosis & 0.00 & 0.00 & 0.27 & 0.00 & 0.00 & 0.20 \\
\hline Lymphoma & 0.00 & 0.41 & 0.12 & 0.24 & 0.00 & 0.07 \\
\hline Demyelinating disease & 0.00 & 0.00 & 0.08 & 0.00 & 0.00 & 0.13 \\
\hline SLE/lupus-like & 0.00 & 0.00 & 0.10 & 0.00 & 0.00 & 0.07 \\
\hline $\mathrm{CHF}$ & 0.00 & 0.00 & 0.28 & 0.00 & 0.00 & 0.00 \\
\hline
\end{tabular}

Abbreviations: CHF, congestive heart failure; SLE, systemic lupus erythematous; PY, patient-years; Ps, psoriasis; PsA, psoriatic arthritis; RA, rheumatoid arthritis; AS, ankylosing spondylitis; JIA, juvenile idiopathic arthritis; CD, Crohn's disease. 
Table 2 Safety and tolerability of long-term adalimumab in randomized controlled trials

\begin{tabular}{|c|c|c|c|c|}
\hline Study & Study design & Treatment arms & Adverse events (\%) & Serious adverse events $(\%, n)$ \\
\hline Gordon et al ${ }^{34}$ & $\begin{array}{l}\text { I2-week double-blind } \\
\text { placebo-controlled } \\
\text { randomized trial in Ps; } \\
\text { open-label extension } \\
\text { until week } 60\end{array}$ & $\begin{array}{l}\text { Adalimumab } \\
40 \text { mg weekly } \\
(n=50) \\
\text { vs } 40 \mathrm{mg} \text { eow } \\
(\mathrm{n}=46) \\
\text { vs placebo } \\
(\mathrm{n}=52)\end{array}$ & $\begin{array}{l}25.2 \% \\
\text { Injection-site pain } 3.4 \% \\
\text { Increased blood } \\
\text { triglycerides } 2.3 \% \\
\text { Nausea } 1.5 \% \\
\text { Dyspepsia } 1.5 \%\end{array}$ & $\begin{array}{l}1.9 \% \\
\text { Malignant melanoma }(n=2) \\
\text { Breast cancer }(n=1) \\
\text { Gastric adenocarcinoma }(n=1) \\
\text { Coccidioidomycosis }(n=1) \\
\text { Recent onset latent } T b(n=1)\end{array}$ \\
\hline Menter et $\mathrm{al}^{35}$ & $\begin{array}{l}\text { I6-week double-blind } \\
\text { placebo-controlled } \\
\text { randomized trial in Ps; } \\
\text { re-randomization of } \\
\text { responders at week 33; } \\
\text { open label extension } \\
\text { until week } 52\end{array}$ & $\begin{array}{l}\text { Adalimumab } \\
40 \mathrm{mg} \text { eow } \\
(\mathrm{n}=8 \mid 4) \\
\text { vs placebo } \\
(\mathrm{n}=398)\end{array}$ & $\begin{array}{l}55.4 \% \\
\text { Injection-site reaction } 3.3 \% \\
\text { Upper respiratory } \\
\text { tract infection } 1.9 \% \\
\text { Nasopharyngitis } 1.4 \% \\
\text { Headache } 1.3 \%\end{array}$ & $\begin{array}{l}0.7 \% \\
\text { Basal cell carcinoma }(n=3) \\
\text { Squamous cell carcinoma }(n=3) \\
\text { Breast cancer }(n=I) \\
\text { Melanoma in situ }(n=1) \\
\text { Tuberculosis }(n=I) \\
\text { Congestive heart failure }(n=1)\end{array}$ \\
\hline Mease et $\mathrm{al}^{41}$ & 24-week double-blind & A dalimumab & Not reported & $0.6 \%$ \\
\hline Gladman et $\mathrm{al}^{42}$ & placebo-controlled & $40 \mathrm{mg}$ eow & Nasopharyngitis $1.8 \%$ & Viral meningitis $(n=I)$ \\
\hline Mease et $\mathrm{a}^{39}$ & $\begin{array}{l}\text { randomized trial in } \\
\text { Ps and PsA; open label } \\
\text { extension study to } 2 \text { years }\end{array}$ & $\begin{array}{l}(n=151) \\
\text { vs placebo } \\
(n=162)\end{array}$ & $\begin{array}{l}\text { Injection-site reaction } 1.2 \% \\
\text { Hypertension } 0.9 \%\end{array}$ & $\begin{array}{l}\text { Myocardial infarction }(n=2) \\
\text { Pulmonary embolism }(n=I) \\
\text { Peritoneal tuberculosis }(n=I) \\
\text { B-cell non-Hodgkin's } \\
\text { lymphoma }(n=I) \\
\text { Non-melanoma skin cancer }(n=3)\end{array}$ \\
\hline
\end{tabular}

Abbreviations: eow, every other week; PS, psoriasis; PsA, psoriatic arthritis; TB, tuberculosis.

ies in PsA, RA, AS, CD, and JIA. Data revealed that the average psoriasis patient treated with adalimumab was 44 years of age, which is 10 years younger than the average RA patient. Also, $61.8 \%$ of RA patients were on concomitant immunosuppressive therapy and $58.6 \%$ were on concomitant systemic steroid therapy compared to only $0.3 \%$ and $1.2 \%$ of psoriasis patients, respectively. Cumulative rates of adverse events in RA patients treated with adalimumab have remained stable over 10 years. Rates of adverse events for psoriasis patients in adalimumab trials were lower compared to those with RA in adalimumab trials, while rates of adverse events for PsA, AS, CD, and JIA were similar to or lower than rates for RA (Table 1). Overall malignancy and mortality rates for adalimumab-treated psoriasis patients were comparable to the general population. ${ }^{40}$

The monthly incidence rate of injection-site reactions in published clinical trials ranged from $1.2 \%{ }^{41}$ to $3.4 \%{ }^{34}$ (Table 3). Within 2 years of exposure to adalimumab, 20/298 patients with PsA discontinued treatment due to adverse events. ${ }^{32}$ Of 3 patients $(1.0 \%)$ who died within the observation period, 2 had myocardial infarction, which was classified as probably not related to adalimumab, and 1 experienced sudden death after acute pulmonary edema, which was classified as possibly related to adalimumab. ${ }^{32}$ No deaths were observed in the other clinical trials included in this systematic review. ${ }^{34,35,43,44}$ Possibly from the associations of co-morbidities with psoriasis and PsA, the randomized clini- cal trial by Genovese et al to assess the efficacy and safety of adalimumab for PsA found significantly more adverse events in patients receiving placebo compared to those receiving adalimumab $(P<0.01){ }^{44}$

Despite the fact that patients were screened for latent $\mathrm{Tb}$ prior to inclusion and not eligible if $\mathrm{Tb}$ was suspected unless $\mathrm{Tb}$ treatment had been initiated already in clinical trials, 3 cases of $\mathrm{Tb}$ were observed in the randomized, placebocontrolled clinical trials (Table 2) ${ }^{34,35}$ Other severe infections observed in clinical trials assessing adalimumab for psoriasis included coccidioidomycosis $(n=1)^{34}$ and viral meningitis $(n=1)^{32,40,41}$ No cases were reported of opportunistic infections in psoriasis patients treated with adalimumab, although a series of cases with Legionella pneumophila pneumonia has been reported in RA patients treated with adalimumab. ${ }^{45}$

Cases of demyelination, optic neuritis, and MS have not been observed in clinical trials of adalimumab for patients with plaque psoriasis or PsA (Table 2). Chung et al report a case of optic neuritis in a psoriasis patient who had been treated for a year with adalimumab (Table 3). ${ }^{46}$ Until prospective registries determine to what extent adalimumab is associated with an increased risk of central nervous demyelination, use of adalimumab should be avoided in patients with a history of MS and patients should be monitored for such early signs of demyelinating disease as weakness in the $\operatorname{limb}(\mathrm{s})$, paresthesias, visual blurring, and ataxia. Through 
Table 3 Case reports of serious adverse events in psoriasis patients treated with long-term adalimumab

\begin{tabular}{|c|c|c|c|c|}
\hline References & $\begin{array}{l}\text { Clinical history } \\
\text { (sex, age, concomitant } \\
\text { medications) }\end{array}$ & $\begin{array}{l}\text { Duration of } \\
\text { adalimumab } \\
\text { prior to SAE }\end{array}$ & SAE & Resolution of symptoms \\
\hline \multirow[t]{2}{*}{$\overline{C h u n g}$ et $\mathrm{al}^{46}$} & $\begin{array}{l}\text { I. Male, age 55, MTX, } \\
\text { simvastatin, atenolol, } \\
\text { aspirin, metformin, } \\
\text { paroxetine, risperidone }\end{array}$ & I.Two months & $\begin{array}{l}\text { I. Rapid painless decrease of } \\
\text { central vision in one eye; } \\
\text { MRI: retrobulbar optic neuritis }\end{array}$ & $\begin{array}{l}\text { I. Complete resolution after } \\
\text { discontinuation of adalimumab }\end{array}$ \\
\hline & 2. Male, age 40 , none & 2. One year & $\begin{array}{l}\text { 2. Progressive visual loss associated } \\
\text { with painful eye movements. } \\
\text { MRI: optic neuritis and numerous } \\
\text { CNS plaques of different ages }\end{array}$ & $\begin{array}{l}\text { 2. Patient continued adalimumab. } \\
\text { Gradual improvement after } \\
\text { steroid treatment }\end{array}$ \\
\hline Deng et $\mathrm{a}^{48}$ & Male, age 54, MTX & One year & $\begin{array}{l}\text { Annular erythematous papules on } \\
\text { thighs and back, clinically and } \\
\text { histologically consistent with } \\
\text { interstitial granulomatous dermatitis }\end{array}$ & $\begin{array}{l}\text { Complete resolution after } \\
\text { discontinuation of adalimumab }\end{array}$ \\
\hline
\end{tabular}

Abbreviations: MTX, methotrexate; SAE, serious adverse event; MRI, magnetic resonance imaging; CNS, central nervous system.

December 2009, no events of progressive multifocal leukoencephalopathy have been reported among subjects participating in controlled clinical trials on adalimumab or in medical published literature.

Because late recurrences of melanoma in RA patients receiving anti-TNF- $\alpha$ treatment have been reported, ${ }^{47}$ patients with a history of melanoma have not been eligible in clinical trials on adalimumab in psoriasis patients. In these doubleblinded, placebo-controlled, randomized clinical trials on psoriasis patients treated with adalimumab, 10 cases of nonmelanoma skin cancers and 3 cases of malignant melanoma were observed (Table 2).

In phase II clinical trials on psoriasis patients treated with adalimumab, a few patients in each treatment group (placebo, $40 \mathrm{mg}$ every other week, $40 \mathrm{mg}$ weekly) converted from negative to positive $(5.8 \%, 2.2 \%$, and $12 \%$, respectively), although a greater number of patients converted from positive to negative ( $8 \%-10 \%)$ during the first 12 weeks of the trial. ${ }^{34}$ The most significant autoantibody induction occurs early in treatment (in the first year) and stabilizes over time. These data (as well as negative to positive shift) suggest that only short-term humoral immune responses are induced during TNF- $\alpha$ blockade. Through December, 2009 , no cases of lupus have been reported in the medical literature after long-term treatment with adalimumab in plaque psoriasis.

Deng et al reported 5 cases of interstitial granulomatous dermatitis developing in patients treated with a variety of TNF- $\alpha$ antagonists, including one patient developing these erythematous annular plaques after treatment with adalimumab for 1 year (Table 3) and which resolved upon discontinuation of adalimumab. ${ }^{48}$
There have also been reports of flaring or changing forms of existing psoriasis as well development of palmoplantar pustulosis in patients with no previous history of psoriasis. ${ }^{49}$ Immuno-histochemical staining of skin biopsy specimens showed increased staining for IFN- $\alpha$ in anti-TNF- $\alpha$-induced psoriasis compared with patients with non-drug induced psoriasis. Collamer et al reviewed all cases of pustular psoriasis occurring during anti-TNF- $\alpha$ therapy and concluded that new-onset psoriasis may occur any time after initiation of anti-TNF- $\alpha$ therapy and often presents with an uncommon morphology or distribution. ${ }^{50}$ They speculated that disruption in cytokine balance following TNF- $\alpha$ inhibition may trigger the upregulation of plasmacytoid dendritic cells and the subsequent production of unopposed interferon- $\alpha$. Multiple reports on the induction or exacerbation of psoriasis in patients treated with TNF- $\alpha$ antagonists exist, ${ }^{51}$ including such cases associated with adalimumab as new onset generalized pustular psoriasis and ${ }^{52}$ pustular psoriasis of the scalp. ${ }^{51}$ Although it has been reported to cause pustular psoriasis, adalimumab has also been found to be effective in the treatment of recalcitrant generalized pustular psoriasis in adults ${ }^{53}$ and adolescents. ${ }^{54}$ Therefore, adalimumab-induced psoriasis requires discontinuation of adalimumab only in cases unresponsive to topical anti-psoriatic treatment.

\section{Conclusion}

Adalimumab has demonstrated excellent efficacy in moderate to severe plaque psoriasis. Based on the current literature on the use of adalimumab for plaque psoriasis, no significant increase in risk of serious infections is observed, in contrast to what has been previously reported in the treatment of RA. Due to differences in co-morbidities, adalimumab treatment 
for patients with moderate to severe plaque psoriasis may have less adverse effects compared to RA patients. Prior to initiation of adalimumab therapy, however, patients should be evaluated for active/latent tuberculosis, serious infections, and other contraindications.

\section{Disclosures}

The authors declare no conflicts of interest.

\section{References}

1. Kurd SK, Gelfand JM. The prevalence of previously diagnosed and undiagnosed psoriasis in US adults: Results from NHANES 2003-2004. J Am Acad Dermatol. 2009;60:218-224.

2. Pariser DM, Bagel J, Gelfand J, et al. National Psoriasis Foundation Clinical Consensus on Disease Severity. Arch Dermatol. 2007;143:239-242.

3. Gladman D, Antoni C, Mease P, Clegg DO, Nash P. Psoriatic arthritis: epidemiology, clinical features, course, and outcome. Ann Rheum Dis. 2005;64:ii14-ii17.

4. Tzu J, Kerdel F. From conventional to cutting edge: the new era of biologics in the treatment of psoriasis. Dermatol Ther. 2008;21: 131-141.

5. Gelfand JM, Shin DB, Neimann AL, Wang X, Margolis DJ, Troxel AB. The risk of lymphoma in patients with psoriasis. $J$ Invest Dermatol. 2006;126:2194-2201.

6. Gelfand JM, Neimann AL, Shin DB, et al. Risk of myocardial infarction in patients with psoriasis. JAMA. 2006;296:1735-1741.

7. Kremers HM, McEvoy MT, Dann FJ, et al. Heart disease in psoriasis. J Am Acad Dermatol. 2007;57:347-354.

8. Gelfand JM, Troxel AB, Lewis JD, et al. The risk of mortality in patients with psoriasis: results from a population-based study. Arch Dermatol. 2007;143:1493-1499.

9. Schmitt JM, Ford DE. Role of depression in quality of life for patients with psoriasis. Dermatology. 2007;215:17-27.

10. Alwawi E, Mehlis S, Gordon K. Treating psoriasis with adalimumab. Ther Clin Risk Manag. 2008;4:345-451.

11. Blauvelt A. T-Helper 17 cells in psoriatic plaques and additional genetic links between IL-23 and psoriasis. J Investig Dermatol. 2008;128:1064-1066.

12. Papoutsaki M, Chimenti MS, Costanzo A. Adalimumab for severe psoriasis and psoriatic arthritis: An open-label study in 30 patients previously treated with other biologics. J Am Acad Dermatol. 2007;57: 269-275.

13. Nestorov I. Clinical pharmacokinetics of TNF antagonists: how do they differ? Semin Arthritis Rheum. 2005;34:12-18.

14. Menter A, Gottlieb A. Guidelines of care for the management of psoriasis and psoriatic arthritis. J Am Acad Dermatol. 2008;58: 826-850.

15. Granneman RG, Zhang Y, Noertersheuser PA, Velagapudi RB, Awni WM, Locke CS, et al. Pharmacokinetic/pharmacodynamic (PK/PD) relationships of adalimumab (HUMIRA, Abbott) in rheumatoid arthritis (RA) patients during phase II/III clinical trials. Arthritis Rheum. 2003;48:S140-S141.

16. Traczewski P, Rudnicka L. Adalimumab in dermatology. Br J Clin Pharmacol. 2008;66:618-625.

17. Kaine J, et al. Effect of adalimumab (HUMIRA ${ }^{\circledR}$ ) on response to pneumococcal and influenza virus vaccines in patients with rheumatoid arthritis (RA). [poster] EULAR, 2006.

18. Johnson D, Jones K, Chambers C. Pregnancy outcomes in women exposed to adalimumab. University of California, San Diego, La Jolla, CA. [abstract] 2009 Annual Meeting of the Canadian Digestive Disease Week (CDDW) held jointly with the 5th Annual Meeting of the Canadian Association for the Study of the Liver (CASF); February 27 March 2, 2009:Abstract 34. Available at: Medical Intelligence Solutions 5198208.pdf.
19. Carter JD, Ladhani A, Ricca LR, Valeriano J, Vasey FB. A safety assessment of tumor necrosis factor antagonists during pregnancy: a review of the Food and Drug Administration Database. J Rheumatol. 2009;36:635-641.

20. Saraceno R, Chimenti, S. How to manage infections in the era of biologics? Dermatol Ther. 2008;8:180-186.

21. Baecklund E, Iliadou A, Askling J, et al. Association of chronic inflammation, not its treatment, with increased lymphoma risk in rheumatoid arthritis. Arthritis Rheum. 2006;54:692-701.

22. Listing J, Strangfeld A, Kekow J, et al. Does tumor necrosis factor alpha inhibition promote or prevent heart failure in patients with rheumatoid arthritis? Arthritis Rheum. 2008;58:637-640.

23. Sarzi-Puttini P, Atzeni F, Shoenfeld Y, Ferraccioli G. TNF-alpha, rheumatoid arthritis, and heart failure: a rheumatological dilemma. Autoimmun Rev. 2005;4:153-161.

24. Domm S, Cinatl J, Mrowietz U. The impact of treatment with tumor necrosis factor- $\alpha$ antagonists on the curse of chronic viral infections: a review of the literature. Br J Dermatol. 2008;159: $1217-1228$.

25. Flendries M, Vissers W, Creemers M, De Jong E, Van de Kerkhof P, Van Riel P. Dermatological conditions during TNF- $\alpha$-blocking therapy in patients with rheumatoid arthritis: a prospective study. Arthritis Res Ther. 2005;7:R666-R676.

26. Eriksson C, Engstrand S, Sundqvist KG. Rantapaa-Dahlqvist. Autoantibody formation in patients with rheumatoid arthritis treated with anti-TNFo. Ann Rheum Dis. 2005;64:403-407.

27. Schiff MH, Burmester GR, Kent JD, Pangan AL, Kupper H, et al. Safety analyses of adalimumab (HUMIRA) in global clinical trials and US postmarketing. Ann Rheum Dis. 2006;65:889-894.

28. Ledington J, Deighton C; on behalf of the BSR. Update on the British Society for Rheumatology guidelines for prescribing TNF $\alpha$ blockers in adults with rheumatoid arthritis (update of previous guidelines of April 2001). Rheumatology. 2005;44:157-163.

29. Van Rijthoven AW, Bijlsma JW, Canninga-van Dijk M, Derksen RH, van Roon JA. Onset of systemic lupus erythematosus after conversion of infliximab to adalimumab treatment in rheumatoid arthritis with a pre-existing anti-dsDNA antibody level. Rheumatology. 2006;45: 1317-1319.

30. Spillane AP, Xia Y, Sniezek PJ. Drug-induced lupus erythematosus in a patient treated with adalimumab. $J$ Am Acad Dermatol. 2007; S114-S115.

31. Sheth N, Greenblatt D, Patel S, Acland K. Adalimumab-induced cutaneous lupus. Clin Exp Dermatol. 2007;32:593-594.

32. Mease PJ, Ory P, Sharp JT, et al. Adalimumab for long-term treatment of psoriatic arthritis: 2-year data from the Adalimumab Effectiveness in Psoriatic Arthritis Trial (ADEPT). Ann Rheum Dis. 2009;68: 702-709.

33. Heiberg MS, Koldingsnes W, Mikkelsen K, et al. The comparative one-year performance of anti-tumor necrosis factor alpha drugs in patients with rheumatoid arthritis, psoriatic arthritis, and ankylosing spondylitis: results from a longitudinal, observational, multicenter study. Arthritis Rheum. 2008;59:234-240.

34. Gordon KB, Langley RG, Leonardi $\mathrm{C}$, et al. Clinical response to adalimumab treatment in patients with moderate to severe psoriasis: double-blind, randomized controlled trial and open-label extension study. J Am Acad Dermatol. 2006;55:598-606.

35. Menter A, Tyring SK, Gordon K, et al. Adalimumab therapy for moderate to severe psoriasis: A randomized, controlled phase III trial. $J \mathrm{Am}$ Acad Dermatol. 2008;58:106-115.

36. Van L, Modi SV, Yang DJ, Hsu S. Sustained efficacy and safety of adalimumab in psoriasis treatment: a retrospective study of 49 patients with and without a history of TNF-alpha antagonist treatment. Arch Dermatol. 2008;144:804-806.

37. Schmitt J, Zhang Z, Wozel G, Meurer M, Kirch W. Efficacy and tolerability of biologic and nonbiologic systemic treatments for moderate-to-severe psoriasis: meta-analysis of randomized controlled trials. Br J Dermatol. 2008;159:513-526. 
38. Gordon KB, et al. Efficacy and safety results in patients with psoriasis treated continuously with adalimumab for 100 weeks. 2008 Fall Clinical Dermatology Conference, Oct 16-19, 2008; Las Vegas.

39. Mease PJ, et al. Sustained clinical efficacy and safety of adalimumab in patients with moderate to severe psoriatic arthritis: 2 years result from an open label extension study. Presented at Winter AAD Meeting, 2008; San Antonio, TX.

40. Burmester GR, Mease PJ, Dijkmans BA, et al. Adalimumab safety and mortality rates from global clinical trials of six immune-mediated inflammatory diseases. Ann Rheum Dis. 2009;68: 1863-1869.

41. Mease PJ, Gladman DD, Ritchlin CT, et al. Adalimumab for the treatment of patients with moderately to severely active psoriatic arthritis: results of a double-blind, randomized, placebo-controlled trial. Arthritis Rheum. 2005;52:3279-3289.

42. Gladman DD, Mease PJ, Ritchlin CT, et al. Adalimumab for long-term treatment of psoriatic arthritis: forty-eight week data from the adalimumab effectiveness in psoriatic arthritis trial. Arthritis Rheum. 2007;56:476-488.

43. Saurat JH, Stingl G, Dubertret L, et al. Efficacy and safety results from the randomized controlled comparative study of adalimumab vs methotrexate vs. placebo in patients with psoriasis (CHAMPION). $\mathrm{Br}$ J Dermatol. 2008;158:558-566.

44. Genovese MC, Mease PJ, Thomson GT, et al. Safety and efficacy of adalimumab in treatment of patients with psoriatic arthritis who had failed disease modifying antirheumatic drug therapy. J Rheumatol. 2007;34:1040-1050.

45. Tubach F, Ravaud P, Salmon-Ceron D, et al. Emergence of Legionella pneumophila pneumonia in patients receiving tumor necrosis factor-alpha antagonists. Clin Infect Dis. 2006;43:e95-e100.
46. Chung JH, Van Stavern GP, Frohman LP, et al. Adalimumab-associated optic neuritis. J Neurol Sci. 2006;244:133-136.

47. Fulchiero GJ Jr, Salvaggio H, Drabick JJ, et al. Eruptive latent metastatic melanomas after initiation of antitumor necrosis factor therapies. $J \mathrm{Am}$ Acad Dermatol. 2007;56:S65-S67.

48. Deng A, Harvey V, Sina B, et al. Interstitial granulomatous dermatitis associated with the use of tumor necrosis factor alpha inhibitors. Arch Dermatol. 2006;142:198-202.

49. Goiiz R, Daudén E, Peréz-Gala S, Guhl G, Garcıa-Diez A. Flare and change of psoriasis morphology during the course of treatment with tumor necrosis factor blockers. Clin Exp Dermatol. 2007;32:176-179.

50. Collamer AN, Guerrero KT, Henning JS, et al. Psoriatic skin lesions induced by tumor necrosis factor antagonist therapy: a literature review and potential mechanisms of action. Arthritis Rheum. 2008;59:996-1001.

51. Wollina U, Hansel G, Koch A, et al. Tumor necrosis factor-alpha inhibitor-induced psoriasis or psoriasiform exanthemata: first 120 cases from the literature including a series of six new patients. Am J Clin Dermatol. 2008;9:1-14.

52. Dalmau J, Roe E, Corella F, et al. Acute generalized skin eruption due to adalimumab: report of two cases. J Eur Acad Dermatol Venereol. 2007;21:1105-1106.

53. Zangrilli A, Papoutsaki M, Talamonti M, et al. Long-term efficacy of adalimumab in generalized pustular psoriasis. $J$ Dermatolog Treat. 2008;19:185-187.

54. Callen JP, Jackson JH. Adalimumab effectively controlled recalcitrant generalized pustular psoriasis in an adolescent. $J$ Dermatolog Treat. $2005 ; 16: 350-352$
Clinical, Cosmetic and Investigational Dermatology

\section{Publish your work in this journal}

Clinical, Cosmetic and Investigational Dermatology is an international, peer-reviewed, open access, online journal that focuses on the latest clinical and experimental research in all aspects of skin disease and cosmetic interventions. All areas of dermatology will be covered; contributions will be welcomed from all clinicians and

\section{Dovepress}

basic science researchers globally. This journal is indexed on CAS The manuscript management system is completely online and includes a very quick and fair peer-review system, which is all easy to use. Visit http://www.dovepress.com/testimonials.php to read real quotes from published authors. 